\title{
Enhancing Bodily Movements of the Visually Impaired Children by Airflow
}

\author{
Fang-Lin Chao ${ }^{1, *}$, Hung-Chi Chu ${ }^{2}$, Liza Lee $^{3}$ \\ ${ }^{1}$ Department of Industrial Design, Chaoyang University of Technology, Chaoyang University of Technology, 436, Taiwan R.O.C. \\ ${ }^{2}$ Department of Information and Communication Engineering, Chaoyang University of Technology, 436, Taiwan R.O.C. \\ ${ }^{3}$ Department of Early Childhood Development \& Education, Chaoyang University of Technology, 436, Taiwan R.O.C.
}

\section{A R T I C L E I N F O}

Article history:

Received: 22 June, 2019

Accepted: 02 August, 2019

Online: 07 August, 2019

\section{Keywords:}

Airflow

Interaction

Bodily movement

Visually impaired

\begin{abstract}
A B S T R A C T
This study involved three departments of teachers and students. The concept of the flowmotivated exercise implemented with a set of flow nozzles. A computer-controlled airflow with specific music introduced, and the motion responses from the participants were analyzed during the initial test and field study. During the initial examination, we adjusted the magnitude and distance setting of the flow and allowed the children to grab the air without music. Structural design, magnetic valve, and interface were implemented based on the insights from the initial test. The redesign performed in a sitting position to reduce the need for students to deviate from the location and fit for physical disabilities during exercise. Four times of visually impaired students were arranged to observe the changes in the movements of students after being guided by the airflow during the teaching process. The performance of the fourth week is better than the previous three weeks. Almost all blind children can make movements in the right position. The cross-domain cooperation experience was beneficial to the facility design.
\end{abstract}

\section{Introduction}

Most of the visually impaired education is established using state funds, and many subjects are integrated with the existing school to incorporate them into society. There are not many schools for the visually impaired students in Taiwan, and some children attend the school for the mainstream setting. Under the guidance of special education teachers, the content of the textbooks for visually impaired students is designed the same as the content for mainstream students.

The core curriculum includes compensatory or functional academic skills, orientation and mobility, social interaction, independent living skills, recreation and leisure skills, technology, and visual efficiency skills [1]. Some activities provided independent in dealing with his environment at a residential school. The students with visual impairment cannot comprehend the entire picture by extracting a lot of information. Visual impairment has complex experience with the spatial world. Study [2] involving fieldwork in everyday life situations to gain insight of their experienced. The allocation of limited instructional hours and the shortage of qualified specialists are significant factors that influence effective instruction.

*Corresponding Author: Fang-Lin Chao, flin@cyut.edu.tw
Experienced teachers are scarce; thus, teaching aid facilities have to be developed on an immediate basis. Teaching aids reduce the problem of repeated guidance and allow students to obtain knowledge in a safe environment. Specialized schools in Taiwan for the visually impaired accommodate students with multiple barriers, such as slow limb movements and cerebral palsy. We observed the activities of these students after class. The students do not have much language communication with each other. When they are bored, they lie on the table or unconsciously tamper with the objects around them. Therefore, the involvement of the management is required. Once the rhythm teaching aids are placed on the table, they are engrossed in playing with the device and have a will to explore (Figure 1). The major mode of teaching is oral instructions. Some students cannot keep up with the progress when they are not attentive. They can explore and feel based on the teacher's instructions by using teaching aids. Rhythm and musicrelated teaching aids help students, especially in terms of instrument appearance- and rhythm-related learning. Learning through teaching aids, which cannot be easily expressed in words, presented its unique benefits.

\subsection{Physical education}

The physical education classes of most students include competitive events, such as running, long jump, or ball-related 


\section{F. Chao et al. / Advances in Science, Technology and Engineering Systems Journal Vol. 4, No. 4, 308-313 (2019)}

games. However, these types of games do not help in enhancing the skills required by the visually impaired students for living. Physical movement and rhythm are necessary skills for the visually impaired students to keep them healthy and to help them obtain higher self-confidence.

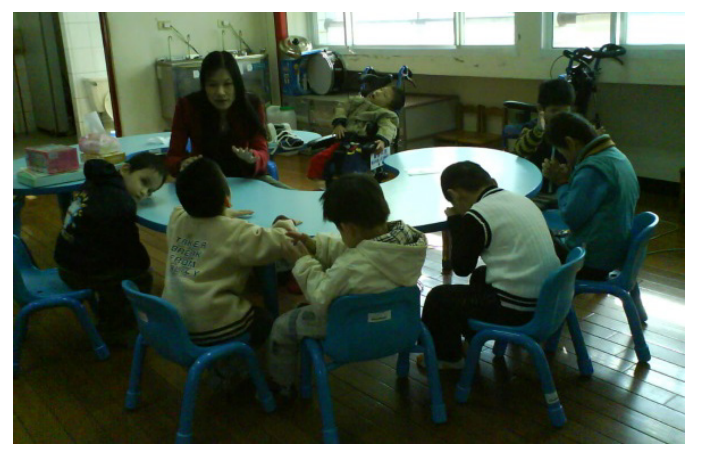

Figure 1: Behavior observation of visually impaired children in an ordinary kindergarten classroom with their teacher [3].

The performance of the visually impaired individuals in physical education is directly associated with the severity of visual impairment, activities performed, the ability to ambulate, and family attitudes [4]. Postmenopausal changes significantly decrease the body's mechanisms for maintaining postural stability. Another study [5] analyzes the effect of sensorimotor exercise on changes in postural stability of visually impaired women. "Late blind persons had a slower walking speed, shorter stride length, and the longer time of gait" [5]. Their gait represents an attempt to adapt to maintain a stable posture during walking.

We must know the students' characteristics (limitations, abilities, and needs) in physical education programs. A school should also be aware of the teaching burden. Visually impaired children face a barrier in being integrated into the general physical education classes [6]. These children display lower physical activity levels because of the lack of instruction and practice.

Teachers sometimes lack sufficient knowledge pertaining to the method of including students. Inadequate preparation, lack of appropriate equipment and curricula, and insufficient teaching hours caused dominant barriers. Consequently, children demonstrated delays in reaching developmental milestones. A sample of 125 visually impaired children was observed [7]. The self-personality and prosocial behavior of the children were explored with respect to their mobility training. The personality well-being and social ability are correlated with the ability to be mobile.

\subsection{Actuator and sensor}

Technology-assisted learning has been a trend in the recent years. People use many apps to explore data, and visually impaired students can expand the scope of activities and overcome physiological limitations through the assistive technology. A study [8] developed smart connected devices for playfulness by using littleBits and Loaded Dice. Authors conducted experiments using visually impaired students and engaged in playful exploration and investigated the extent of providing imaginative design concepts. Another study [9] provided a visually impaired user interface by using embossed two-dimensional patterns that can be read using haptic sensing. In [10], a researcher introduced a haptic device that can notice the presence of obstacles inside an environment. A prototype that was implemented using a short cane and an embedded smart sensing strategy was presented.
The engineers used senses other than the visual sense to transmit a message to the visually impaired. For example, tactile pressure, temperature field, airflow field, and sound can be reconsidered for such applications that are focused on the visually impaired people. When these applications are aimed at the visually impaired population, we must reduce the cost and complexity of the technology because a high price renders the technology unaffordable and a complicated technology cannot be used reliably.

Kraus [11] designed teaching tools that are appropriate for exploring the solar system and different astronomical subjects. The magnetic field, temperature drop, and airflow connector presented physical space for the visually impaired people. The availability of the tactile constellation presents apparent magnitudes and displays the star distribution in the Cassiopeia constellation.

\subsection{Flow-Motivated Interactions}

This study focuses on three considerations: (1) providing haptic attention of airflow, (2) encouraging natural response from children, and (3) engaging to relate the body motion based on the excitation. Although the target users are visually impaired children, ordinary people or the elderly can follow the rhythm and find the pattern to play. The flow-motivated game uses a set of flow nozzles that can generate segmented air flow through a compressor. An air compressor is readily available in a shop. The compressor increases the pressure in the air-intake system [12] and generates a mass flow. The intensity of the mass flow can be sensed by the skin.

The airflow should be sensed by the skin; however, a very strong airflow causes discomfort. A small pipe sends the jet stream, and the high-speed air stream simultaneously causes a concomitant acoustic field with a sharp sound. Moreover, the time required for a child to respond to the airflow by performing actual body action should be considered. The response to the airflow varies from person to person. Some people move in the direction of the airflow, and some people grab the source of the airflow. This design encourages a spontaneous response and does not force the user to respond in specific manner. When a user is not pressurized to act in a specific manner, he or she can easily respond spontaneously.

The third design consideration was engagement to relate the body motion based on the excitation. The stimulation of the airflow cannot be too frequent and unpredictable and within a reasonable reaction time. The rhythmic stimuli are matched with the rhythm of the tones, thus promptly providing airflow guidance while providing the advice using the sounds. We provided four-six airflow spouts at appropriate body parts. The upper body and the lower body have three spouts each. The airflow field of each spout could be felt and was used based on the position of the person standing.

\section{Materials and methods}

At initial test, we used four impingement spouts without music. Teacher controls the gas flow and let the children try to grab the air. The four heads are connected to the air compressor respectively, and we control the opening and closing of the airflow by hand. We need to understand the impact of the height, position, and distance of the nozzle on the child before design. Teacher fixed the spout with a height-adjustable bracket, which was initially located at the waist of the children.

During test the position was adjusted to avoid being touched. Students listen to the teacher's introduction before they start. We 
found that at the beginning, they were a bit nervous and the action was not very casual. The mentality may be a bit like facing the exam, not in a state of play.

\subsection{Initial test}

The experimental design used four of airflow without music. Most subjects stood still in the center without body movement and facial expression at the beginning (Figure 2).

When the airflow came out from the four ventilators at the same time, subject A,C,E,F,G,K,L,M,N,O didn't move at all.

They stood still until the air stopped. (O-A,C,E,F,G K,L,M,N,O -0325-1,2,3,4) (O-A, C, E, F, G, K,L, M, N, O-0325$\mathrm{R})$

When subject K, L, M, N, O felt the airflow, they didn't know how to move because they are too young.(I-0325-T)

A few subjects didn't do any movement but did smile. Subject $\mathrm{B}$ and $\mathrm{D}$ didn't do any bodily movement but a few seconds later, they started smiling. (O-B, D -0325-1, 2, 3, 4) (O-B, D -0325-R)

A few subjects didn't show any expression, but moved their hands. Subject B and D didn't do any bodily movement but a few seconds later, they started smiling. (O-H, I, J -0325-1, 2, 3, 4) (OH, I, J -0325-R)

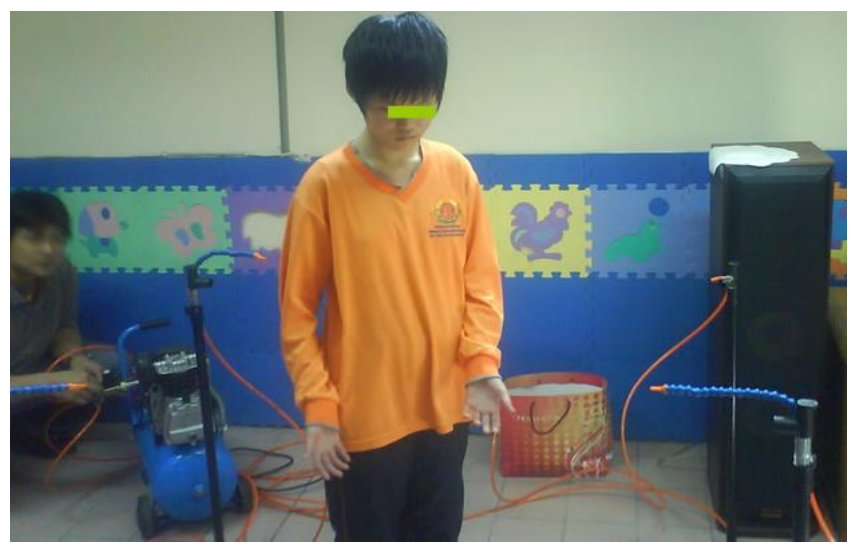

Figure 2: Initial test setup for evaluating how visually impaired children respond to gas spreading

\subsection{Discussion of initial test}

This test revealed some problems.

(1) Some children did not know how to respond and became frustrated. When the students asked "Why am I coming?", they intended to tell the teacher "what should I do in this situation?". Formal learning habits constrain the mood of participation.

(2) Although some children were attracted to the flow in the first few rounds, they became bored because the same motions were repeated. Simple physical movements allowed them to respond only mechanically. The lack of self-inherent motivation reduces their interest.

(3) Some children paid so much attention to others' opinions that they became distracted and began playing. The students should be integrated in the event with a relaxed mood.

(4) The airflow produces a sharp sound. The air flow must be moderate. The motor generated considerable noise when the air pressure of the air compressor reduced. Children can get scared because of the sudden changes in the outside world.

(5) We determined whether a garment blocks airflow and found that the students did not rely solely on the airflow sprayed on the skin. When the airflow ejected, the turbulence air generated a sharp sound. Visually impaired children are sensitive to hearing, and they can determine the source of the airflow based on the noise.

\section{Design of Flow Control}

For realizing a simplistic design and the lowest price for the visually impaired, we avoided the complicated design to reduce possible failures. A central computer with touch panel controls the progress of the song and direction of the gas. Figure 3 displays the structure of the system. In the concept proposal, we suggested that the ground has a pressure sensor to determine the user's position and that infrared sensors can be installed on the bracket to know how the user moves.

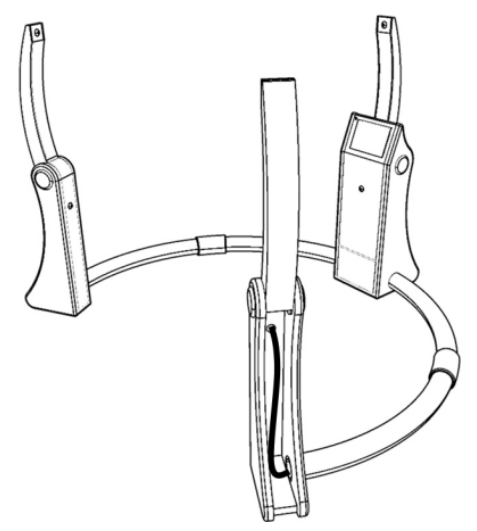

Figure 3: Diagram of the flow-motivated game with a magnetically controlled valve. Based on the problems found from the test model, a setup model with three nozzle arms was selected.

The initial test model was arranged with an air compressor and four nozzles. When the air compressor was switched on, the air reservoir of the air compressor was gradually pressurized. At this time, the control valve was opened to eject air. We used a magnetic valve to control the air outlet switch, which can be controlled remotely using an electrical signal.

The height of the three brackets was set based on the height of the children, and part of the gas was transmitted from above. Thus, the height of the bracket was set at $160 \mathrm{~cm}$ (Figure 3). There is an air outlet at the upper and middle sections of the bracket. The central part contains a rotating shaft, and the top arm can be folded into the lower feet. The circular frame in series was placed on ground to stabilize the three main arms. Control wires and airflow conductors were routed from the central computer through those tubes.

\subsection{Circuit Design}

Prototypes involving the control circuit and user interfacing software were developed. A USB interface control panel and solidstate relay (SSR) were placed on the circuit board (Figure 4). The Phidget Interface Kit [13] was utilized intangible user interfaces; the interface kit screen controller provides accurate results. We used the Phidget Interface 8/8/8 input-output board servers as a communication channel with the host computer. 
The program controls the switching of the gas nozzles by using a preset sequence. The USB interface board cannot provide sufficient electrical current to switch the magnetically controlled valve. We constructed an external power board to ensure adequate current supply. An SSR has neither moving parts nor arcing contacts, which are often the primary cause of failure [14]. This provides a high degree of reliability and a long service life.
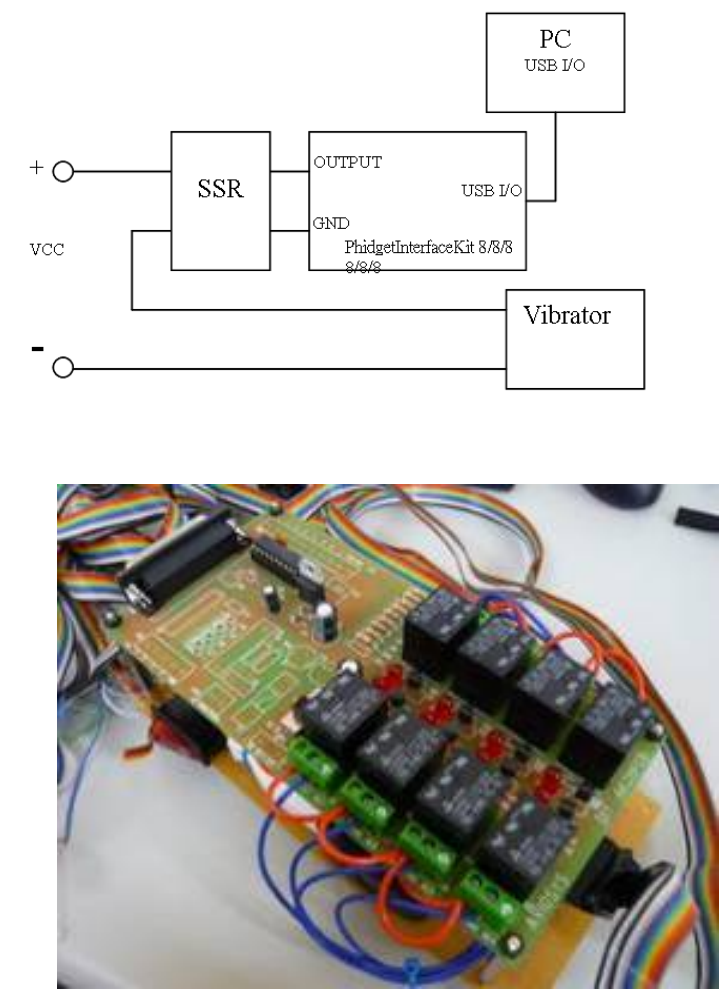

Figure 4: Circuit diagram and SSR circuit board with an external power supply [14].

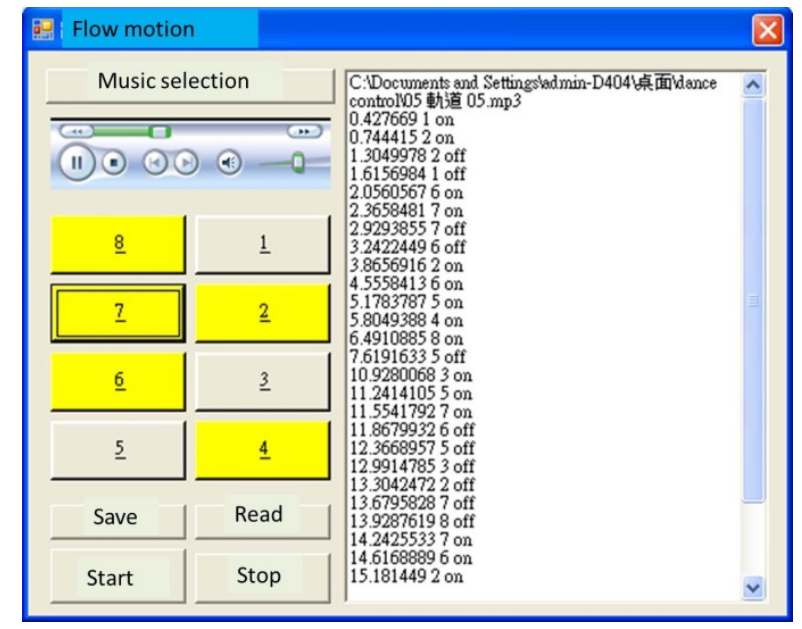

Figure 5: Software interface layout design with a sequential setting of the gas outlet and music control. The yellow blocks indicate active channels.

\subsection{Dancing control editor and graphical user interface}

As displayed in Figure 5, the interface control program has a graphical user interface that was developed on Microsoft Visual Studio 2005. The graphical user interface displays the current state of each switch. The button with the yellow background color indicates that it is currently activated. The text area on the righthand side lists the sequence of the switching actions. A user can manually control the switching by directly clicking on the buttons at the specific time while the music is playing.

The status indicator, file read, and start-stop buttons are above the music editor. We can see the blocks $8,7,6,2$, and 4 in yellow, which implies that five nozzles are switched on. In the subsequent tests, we found that when most of the spouts simultaneously ejected, the students got confused while determining the source.

\subsection{Multidisciplinary}

This study comprises three departments of teachers and students. The Department of Industrial Design is responsible for proposing the structure of the concept. The students of the computer communication department are familiar with the circuits and programs and can construct the control circuit and the software to develop a working model. The students from the Department of Early Childhood Education invited children to try out prototypes in special education schools and found the areas of improvement in trial teaching.

In the past, industrial design paid more attention to the performance of modeling pursuit; computer students' interest in the pursuit of technology, they often prefer multifunctional betting into products. In the course of teaching, we understood that the function of a product is not the main part, and the user is the main part. A teacher has to adopt the story-telling method in the guiding process to allow the users to accept the taught content wholeheartedly. The experience of integrated research can adjust the design parameters and get an appropriate product design.

\section{Field Study Results}

The purpose of the field study was to examine a combination of music and airflow to enhance the bodily movement of visually impaired people. The methodology of this study was based on a qualitative study using interviews with students and the classroom teacher and observation reports from four observers and the researcher. Fifteen 4-19-year-old visually impaired students who enrolled in a school for the visually impaired in Central Taiwan were selected through purposive sampling for the study.

\subsection{Materials and methods}

The assessment instruments included structured observation forms to gather data on the reaction of the subjects from the researcher and four observers who were trained graduate students, interview reports from the teacher and the subjects at school. All interview sessions were recorded on video, and these were viewed and reported by four observers. The following is the coding symbol of the study:

Table 1: Coding symbol for music and flow activities

\begin{tabular}{|c|c|}
\hline Coding & Meaning \\
\hline $\begin{array}{c}\text { O-A- } \\
0325-4\end{array}$ & $\begin{array}{c}\text { Observation of subject A on March 25, } \\
2016 \text { by observer 4 }\end{array}$ \\
\hline $\begin{array}{c}\text { O-B- } \\
\text { 0325-R }\end{array}$ & $\begin{array}{c}\text { Observation of subject B on March 25, } \\
2016 \text { by the researcher }\end{array}$ \\
\hline I- 0325-T & $\begin{array}{c}\text { Interview with the teacher on March 25, } \\
2016\end{array}$ \\
\hline I-C-0325- & $\begin{array}{l}\text { Interview with subject C on March 25, } \\
\text { 2016 by the researcher }\end{array}$ \\
\hline
\end{tabular}


The experimental design utilized four ventilators of airflow simultaneously with music. While revised experimental design from airflow only to play with music simultaneously, some of the subjects smiled but did not do too much movement, especially the five preschoolers. Most subjects' bodily movement was hand movement only.

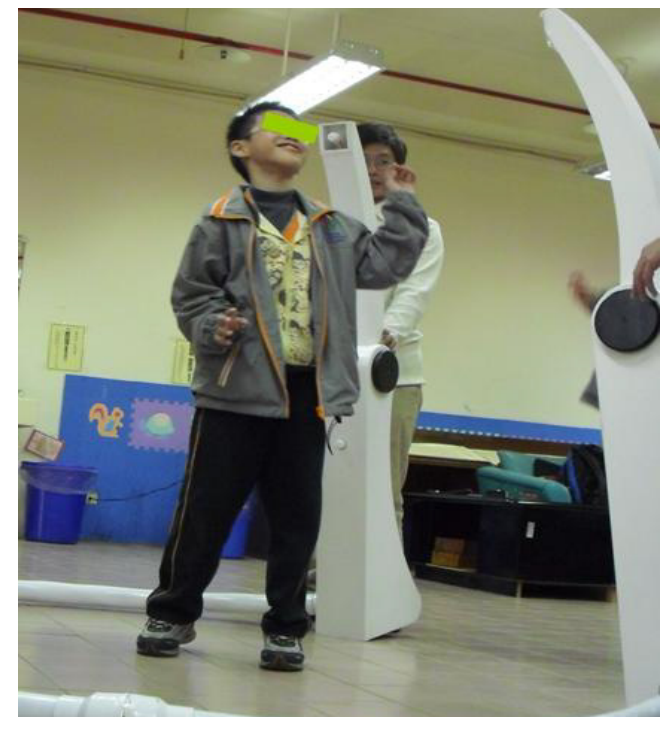

Figure 6: Children interacting with the airflow

\subsection{Results}

In order to facilitate identification, we use the English symbol A B C D... to represent the participating students. While subject A, B, C, D, E, F, G, H, I and J listened to the music and felt the airflow, they didn't have any bodily movement but a few seconds later, they started smiling. (O- A, B, C, D, E, F, G, H, I, J-0415-1, 2, 3, 4) (OA, B, C, D, E, F, G, H, I, J -0415-R)

The younger subject K said "It's music" while listening to the music. He also used his hands to feel the airflow. (O-K-0415-1, 2, 3, 4) (O-K -0415-R)

The subjects $\mathrm{L}, \mathrm{M}, \mathrm{N}$ and $\mathrm{O}$ all moved their hands to feel the airflow while the music played. (O-L, M, N,O-0415-1, 2, 3, 4) (OL, M, N, O -0415-R)

When the researcher asked the subject $\mathrm{K}, \mathrm{L}, \mathrm{M}, \mathrm{N}, \mathrm{O}$ "Do you like music?" they all answered "Like." (O-K, L, M, N,O-0415-1, 2, 3, 4) (O- K, L, M, N, O -0415-R)

The subject $\mathrm{K}$ smiled. He said he liked the music and airflow. (O-K-0415-1, 2, 3, 4) (O-K -0415-R)

Due to the limited movement, the study changed the dynamics of airflow and music. Some subjects did not like the ventilators with strong airflow simultaneously with loud music. Some subjects said it was hard to feel anything from the four ventilators with weak airflow simultaneously with soft music.

When the four switches of airflow were opened, the researcher asked subject K "Would you like to move?" He said "Yes." But subject $\mathrm{K}$ needed to be held with the researcher. He wanted to find the airflow with the researcher. (O-K-0415-1, 2, 3, 4) (O-K -0415$\mathrm{R})$

When the four switches of airflow were opened, subject $\mathrm{L}$ moved his body to face the air and moved his hands to touch the tube, (O-L-0415-1, 2, 3, 4) (O-L -0415-R)
When the four switches of airflow were opened, subject $M$ stuck out his tongue and opened his mouth to feel the air. (O-M0415-1, 2, 3, 4) (O-M -0415-R)

During four ventilators of weak airflow simultaneously with soft music, the subject $\mathrm{O}$ liked it and smiled. (O-O-0415-1, 2, 3, 4) (O-O -0415-R)

When the researcher asked the subject $\mathrm{P}$ "Do you like the air and music?" he said "like." (O-P-0415-1, 2, 3, 4) (O-P -0415-R)

When the ventilators were opened randomly, most older subjects could find the direction to adjust and move their hands and feet to feel it. (O- A, B, C, D, E, F, G, H, I, J -0415-1, 2, 3, 4) (OA, B, C, D, E, F, G, H, I, J -0415-R)

\subsection{Discussion of field test}

The visually impaired children like music; thus, music it could be a good motivator for them to exercise. The study also found that children enjoy catching the flow. Sometimes their hand touched the air outlet, and this disturbed the original setup of the gas flow direction (Figure 6). This free exploration usually occurred in the first class and should be encouraged. With the teacher's guidance, the students gradually responded to such airflow with their body. Giving the students freedom to explore is also an essential factor. When students reflect based on specific rules, they lose their interest. Moreover, the interaction between peers affects an individual's attitudes. Peers' experience of trying can also stimulate the willingness of the students to try.

The results suggest avoiding frequent changes in the direction of the airflow. Students give up when they are unable to respond appropriately. The design was such that the students could be guided using a small amount of airflow from above or below depending on the characteristics of the music. Students become familiar with it by gradual learning.

The sound of the motor is loud when the air compressor is on, which scares the children. One of the solutions is to move the air compressor to another space or use a small air compressor. Then, the air volume ejected by the device can be reduced once the students are familiar with the device.

\section{Redesign}

The redesign adopted sitting position (Figure 7) to reduce the need for students to deviate from the location and fit for physical disabilities during exercise. The air outlet is located at the lower edge of the table to avoid being deflected by the student. Infrared sensors arranged on the side of the air outlet end, and a variable resistor provided for adjusting the distance of infrared emission and reception. Visually impaired students with physical disabilities sit in the middle and shift their bodies with the direction of the airflow; this prevents them from falling due to instability during the shift. Four times of visually impaired students were arranged to observe the changes in the movements of students after being guided by the airflow during the teaching process. There were six subjects under test, and Professor Li was composing music for visually impaired children. The TA will fill in the average performance (1-5 points), range of action, and emotional response of the children.

The first week found that due to the experience of no contact airflow in daily life, there was rejection at the beginning of the test. Subject A needs the teacher to encourage him to find the outlet point actively. Subject B can actively touch after the teacher's 
guidance, but the body does not move when the air jets. After the guides explained, they were able to proactively find the airflow, but the movements did not change much (Figure 7a). The performance of the fourth week is better than the previous three weeks. Almost all blind children can make movements in the right position (Figure $7 \mathrm{~b}$ ) and can feel the way the airflow interacts. The test results show that the overall average performance is increasing from 1.6 to 4.3 . The average number of follow-up airflows was 4.31. In the magnitude of the action (can swing the limb) up to 3.12. When the music and the rhythm of the airflow match, it is more inspiring to the children's movements.

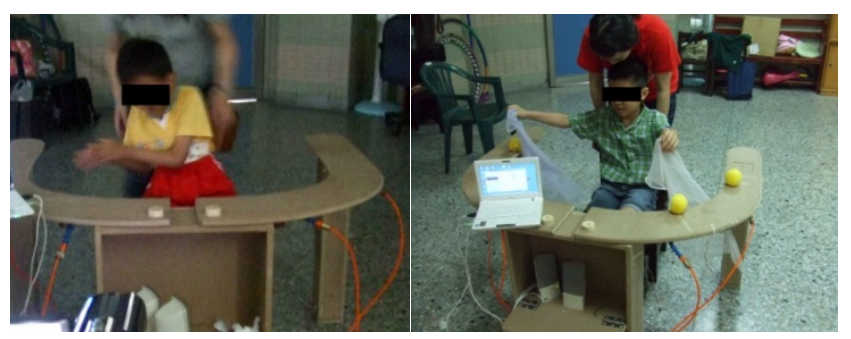

(a)

(b)

Figure 7: Response of the respondent (a) First week, (b) the Fourth week Encourage students to respond with a silk scarf.

\section{Conclusions}

We presented a flow-motivated facility that adopts noncontact techniques for enhancing the exercising behavior. The airflow reinforces active participation of visually impaired children and increases positive interactions. The results of the study revealed that visually impaired children like music; thus, music could be a good motivator for them to exercise. They were beneficial to develop more holistic equipment for the visually impaired. Brief comparisons of the results against previous findings in [4] and [6], the decreasing of the body's mechanisms for maintaining postural stability can gradually improve after systematic learning and stimulation. Children enjoy catching the airflow; thus, sometimes their hand touched the air outlet and disturbed the original setup of gas flow direction. The study comprised three departments of teachers and students. This cross-domain cooperation experience was quite rare for us. Appropriate guidance during the teaching process is vital for the user to accept the product. The integrated research can adjust the design parameters and obtain a proper product design. The use of appropriate technology solves user problems and reduces the user's frustration.

\section{References}

[1] Hatlen, P., "The core curriculum for blind and visually impaired students, including those with additional disabilities" Review 28(1), 25-32, 1996.

[2] Cecilie H. Anvik, "Embodied spaces in the making: visually impaired people, bodies and surroundings" Scandinavian Journal of Disability Research, 11(2), 145-157, 2009. DOI: 10.1080/15017410902830710

[3] Chao, F.L., Lee, L., Chu, H.C. \& Wu, W.T., "A Pilot Study on Applying Combination of Music and Airflow to Enhance Bodily Movement of Visually Impaired" in P. Resta (Ed.), Proceedings of Society for Information Technology \& Teacher Education International Conference, 3564-3569, Austin, Texas, USA, 2012.

[4] Winnick, J.P., "The performance of visually impaired youngsters in physical education activities: Implications for mainstreaming" Adapted Physical Activity Quarterly, 2(4), 292-299, 1985.

[5] Zuzanna Maćkowiak, Wieslaw Osiński,"The Effect of Sensorimotor Training on the Postural Stability of Visually Impaired Women Over 50 Years of Age" Journal of Women \& Aging, 27(1), 68-80, 2015. DOI: $10.1080 / 08952841.2014 .928140$
[6] Wiskochil, B. et al. "The effects of trained peer tutors on the physical education of children who are visually impaired" Journal of Visual Impairment \& Blindness, 101(6), 339-350, 2007.

[7] Malik, S. et al., "Orientation and mobility training in special education curriculum for social adjustment problems of visually impaired children in Pakistan" International Journal of Instruction, 11(2), 185-202, 2018.

[8] Bischof, A. et al. "Exploring the playfulness of tools for co-designing smart connected devices: a case study with blind and visually impaired students" Proceedings of the 2016 Annual Symposium on Computer-Human Interaction in Play Companion,. ACM, 2016.

[9] Lefeuvre, K. et al. "Loaded dice: exploring the design space of connected devices with blind and visually impaired people" Proceedings of the 9th Nordic Conference on Human-Computer Interaction, ACM 2016.

[10] Ando, B. et al. "A haptic solution to assist visually impaired in mobility tasks" IEEE Transactions on Human-Machine Systems, 45(5), 641-646, 2015.

[11] Kraus, S. "Astronomy for the blind and visually impaired" Bringing the Universe to the World, 36, 2016.

[12] Gibby, D. G. U.S. Patent No. 9,133,759. Washington, DC: U.S. Patent and Trademark Office. 2015.

[13] Bagwan, F. et al. "Tangible user interface based descriptor for appliances" National Conference NCPCI, 2016.

[14] Verma, N., Gupta K., \& Mahapatra S. "Implementation of solid state relays for power system protection" International Journal of Scientific \& Technology Research 4(6), 65-70, 2015. 